ISSN 0103-5150

Fisioter. Mov., Curitiba, v. 24, n. 1, p. 133-140, jan./mar. 2011 Licenciado sob uma Licença Creative Commons

\title{
Eficácia de um protocolo de exercícios físicos em pacientes com insuficiência renal crônica, durante o tratamento de hemodiálise, avaliada pelo SF-36
}

\author{
Efficacy of a physical exercises protocol in patients with chronic \\ renal failure during treatment of hemodialysis, valued by SF-36
}

\author{
Karoline Teles de Araújo Soares ${ }^{[a]}$, Marcel Vidal Viesser ${ }^{[b]}$, Tânia Aparecida Barbosa Rzniski ${ }^{[c]}$, \\ Edison Paula Brum ${ }^{[d]}$ \\ [a] Fisioterapeuta, Especialista em Dérmato Funcional pela Universidade Positivo (UP), Curitiba, PR - Brasil, e-mail: \\ karolsoares@hotmail.com \\ [b] Fisioterapeuta pela Universidade Positivo (UP), Curitiba, PR - Brasil, e-mail: marcel.viesser@gmail.com \\ [c] Fisioterapeuta, Mestra em Gestão Ambiental, professora adjunto do curso de Fisioterapia e orientadora do estudo da \\ Universidade Positivo (UP), Curitiba, PR - Brasil, e-mail: tb.rzniski@up.edu.br \\ [d] Médico, Especialista em Nefrologia, professor adjunto do curso de Medicina da Universidade Positivo (UP), Curitiba, PR - \\ Brasil, e-mail: brum@up.edu.br
}

\section{Resumo}

Objetivo: Analisar os efeitos de um protocolo de exercícios fisioterapêuticos em pacientes renais crônicos, durante a terapia hemodialítica, visando à melhora de sua qualidade de vida. Materiais e métodos: Foram avaliados 27 pacientes, com idade média de $51 \pm 10,5$ anos, índice de massa corpórea média de 24,3 $\pm 3,8$ e em tratamento hemodialítico há aproximadamente $50 \pm 27,7$ meses. Os pacientes assinaram o Termo de Consentimento Livre e Esclarecido e, durante três meses, participaram de um programa de tratamento, durante a hemodiálise, composto por alongamentos musculares de membros inferiores, de membro superior (que não apresentasse a fístula arteriovenosa), lombar e cervical (caso o paciente não apresentasse o cateter cervical de diálise); fortalecimentos musculares de membros inferiores e membro superior, sem o acesso vascular; e relaxamento. Foi aplicado o questionário de qualidade de vida SF-36 antes e após o período de tratamento. Resultados: Após o tratamento, o teste SF-36 mostrou melhora significativa das seguintes variáveis: capacidade funcional, nível de dor, vitalidade e saúde mental. Também foi observado que, antes do tratamento, dez pacientes relatavam cãibras musculares e, após a fisioterapia, somente quatro pacientes 
continuaram relatando essas contrações. Conclusão: Conclui-se que a atuação da fisioterapia durante a hemodiálise contribuiu para a melhora da qualidade de vida de pacientes renais crônicos.

Palavras-chave: Insuficiência renal crônica. Hemodiálise. Fisioterapia.

\section{Abstract}

Objetctive: To analyze the effects of a physiotherapeutic exercises protocol in chronic renal patients during hemodialysis therapy, aiming at improve their quality of life. Materials and methods: We evaluated 27 patients, mean age $51 \pm 10.5$ years, mean body mass index of $24.3 \pm 3.8$ and submitted to hemodialysis treatment for approximately $50 \pm 27.7$ months. All patients signed a written informed consent form and, for three months, participated in a program of physiotherapy treatment during hemodialysis, composed by muscle stretching of lower limbs, upper limb (opposite to the arteriovenous fistula), lumbar and cervical muscle stretching (if patient did not present cervical catheter for dialysis); muscle strengthening of lower limbs and upper limb without vascular access, and relaxation. Medical Outcomes Study 36 - Item Short Form Healthy Survey (SF-36) was applied before and after treatment. Results: After treatment, SF-36 test showed significant improvement of the following variables: functional capacity, level of pain, vitality and mental health. It was also observed that, before treatment, 10 patients reported muscle cramps, and after therapy, only 4 patients remained with this symptom. Conclusion: The performance of the physiotherapy during hemodialysis contributed to improve quality of life of chronic renal patients.

Keywords: Chronic renal insufficiency. Hemodialysis. Physiotherapy.

\section{Introdução}

A insuficiência renal crônica (IRC) é definida como uma síndrome caracterizada pela perda lenta, progressiva e irreversível da capacidade excretória renal (1-4). A incidência da dessa síndrome tem aumentado progressivamente a cada ano em todo o mundo, o que representa elevados custos para manter os pacientes em terapia renal substitutiva (TRS) (5). 0 Censo da Sociedade Brasileira de Nefrologia de 2007 indicou que, em janeiro daquele ano, a prevalência de pacientes em diálise por milhão da população (pmp) era de 73.605 (391 pacientes pmp) (6). Por isso, a IRC é caracterizada como um grave problema de saúde pública (1, 7-9).

A IRC é multicausal, apresentando diversos fatores de risco. De acordo com inquéritos realizados pela Sociedade Brasileira de Nefrologia, no período de 1996/1997, as principais doenças diagnosticadas como sendo potenciais causas de IRC são: a hipertensão arterial sistêmica ( $24 \%$ dos casos), a glomerulonefrite $(24 \%)$ e diabetes mellitus $(17 \%)(3,4)$. Outras causas devem ser consideradas, como: pielonefrite, doença policística renal, doenças autoimunes, etc $(2,6,10)$.

Nas fases iniciais da IRC, o paciente pode não apresentar manifestações clínicas, porém, a perda progressiva da função renal vai desencadear múltiplos sinais e sintomas para o paciente (2), afetando quase todos os seus sistemas orgânicos (11-13). Dentre esses sintomas, podemos citar: hiperpotassemia, acidose metabólica, hipocalcemia, edema periférico, insuficiência cardíaca congestiva, cãibras, fraqueza muscular, etc $(1-3,11)$.

Os tratamentos disponíveis para a IRC são a diálise peritoneal ambulatorial contínua (DPAC), a diálise peritoneal automatizada (DPA), a diálise peritoneal intermitente (DPI), o transplante renal (TX) e a hemodiálise (HD) (8). Deve-se frisar, porém, que todos os tratamentos acima descritos não curam a doença, apenas substituem parcialmente a função renal (8).

Nos últimos anos, a hemodiálise tem sofrido importantes mudanças e inovações tecnológicas (10). Martins, Vieira e Bras $(8,14-16)$ relatam a melhora da sobrevida dos pacientes em hemodiálise, embora com prejuízo em sua qualidade de vida relacionada à saúde (QVRS). Lima e Santos $(10,17)$ afirmam o oposto, ou seja, não houve incremento na sobrevida desses pacientes.

A QVRS dos pacientes com IRC em tratamento hemodialítico está ligada a diversos fatores, sendo um deles a idade. Vários estudos (16-19) indicam o 
aumento da idade média dos pacientes que iniciaram a hemodiálise nos últimos anos, o que tem relação direta com a perda de capacidade funcional, a vitalidade e os aspectos sociais do sujeito.

A QVRS desses indivíduos também está diretamente ligada às complicações que eles podem vir a apresentar. Muitos autores $(9,15,16,20-22)$ haviam apontado para uma série de distúrbios osteomioarticulares crônicos, relacionados ao metabolismo ósseo, que o paciente portador de IRC pode vir a apresentar, alguns de particular interesse para a fisioterapia.

Henn (23) afirmou que o paciente renal crônico apresenta excesso de líquidos corporais que deixam os órgãos congestos, inclusive os pulmões. A tolerância do portador de IRC ao exercício fica gravemente reduzida $(14,24)$. Em torno de $50 \%$ dos pacientes vão a óbito por complicações cardíacas (20).

Outros fatores que certamente levam à deterioração da QVRS dos portadores de IRC em programa de hemodiálise são: o convívio com uma doença incurável, o esquema rigoroso da terapêutica (que provoca fortes modificações nos hábitos alimentares, nas atividades sociais e no trabalho), a utilização de vários medicamentos e a dependência de uma máquina $(17,25)$.

A literatura pouco fala sobre a atuação da fisioterapia em pacientes renais crônicos durante o regime de hemodiálise. Peres e Reboredo $(25,26)$ indicam que um programa de exercícios físicos durante a diálise promove melhora da capacidade funcional, da força e resistência muscular, da função cardíaca e, consequentemente, da qualidade de vida. Soares et al. (27) afirmam que exercícios físicos durante a diálise são uma estratégia eficiente para dar motivação aos pacientes em um ambiente estruturado e monótono. Por isso, o objetivo da seguinte pesquisa é analisar, por meio do SF-36, os efeitos que um protocolo fisioterapêutico pode proporcionar aos pacientes renais crônicos durante a terapia hemodialítica, visando à melhora de sua qualidade de vida.

\section{Materiais e métodos}

Este estudo é do tipo experimental, longitudinal e com abordagem quantitativa, sendo realizado no Instituto do Rim do Paraná, no período noturno, e tendo aprovação do Comitê de Ética em Pesquisa do Centro Universitário Positivo (n. 94/2007).
Os critérios de inclusão foram os seguintes:

- idade entre 40 e 60 anos;

- tempo de hemodiálise acima de 12 meses;

- ausência de déficit cognitivo;

- aceitação para participar da pesquisa, mediante a assinatura do Termo de Consentimento Livre e Esclarecido.

Já o critério de exclusão foi:

- não entendimento do Medical Outcomes Study 36 - Item Short Form Health Survey (SF-36).

A amostra inicial era constituída por 46 pacientes, porém, cinco recusaram-se a participar da pesquisa, dois mudaram de turno, dois desistiram da pesquisa, um saiu da instituição, um realizou transplante renal e oito não se enquadraram nos critérios de inclusão. Portanto, a amostra final foi composta por 27 pacientes.

Inicialmente, os pacientes foram instruídos quanto aos métodos empregados na pesquisa e, caso aceitassem participar, assinavam o Termo de Consentimento Livre e Esclarecido. Em seguida, preencheram o Medical Outcomes Study 36 - Item Short Form Health Survey (SF-36), sendo este um instrumento genérico, de fácil administração e compreensão (26), que avalia, de forma quantitativa, o nível de qualidade de vida dos pacientes, levando em consideração as seguintes dimensões: capacidade funcional (desempenho nas AVDs - atividades de vida diária); aspectos físicos (impacto da saúde física nas AVDs e nas atividades profissionais); dor (nível de dor e impacto sobre as AVDs e as atividades profissionais); estado geral de saúde (percepção subjetiva do estado geral de saúde); vitalidade (percepção subjetiva do estado de saúde); aspectos sociais (influência da saúde física sobre as atividades sociais); aspectos emocionais (influência da condição emocional sobre as AVDs e as atividades profissionais); e saúde mental (escala de humor e bem-estar) (6, 8, $17,18,27,28$ ). A pontuação varia de 0 (pior resultado) a 100 (melhor resultado) $(6,8,17,18,27,28)$.

A partir da semana seguinte, eles participaram de um programa de tratamento fisioterapêutico composto por: alongamentos musculares de membros inferiores, de membro superior (que não apresentasse a fístula arteriovenosa), lombar e cervical (caso o paciente não apresentasse o cateter cervical de 
diálise); fortalecimentos musculares de membros inferiores e membro superior, sem o acesso vascular (a carga e as repetições eram predeterminadas, porém, adaptáveis à capacidade do paciente); e relaxamento, envolvendo a conscientização respiratória.

0 protocolo fisioterapêutico foi individualizado para cada paciente, respeitando seus limites e tendo duração de, aproximadamente, 25 a 30 minutos. Os exercícios terapêuticos sempre foram realizados em torno de meia hora após o início da hemodiálise, respeitando a condição clínica do paciente. A pressão arterial (PA) e a frequência cardíaca (FC) sempre eram aferidas antes e após a terapia. 0 paciente era dispensado da fisioterapia quando apresentava quadro álgico muito importante, dispneia, tonturas, hipertensão ou hipotensão acentuadas.

Os pacientes foram atendidos duas vezes por semana, no período de junho a setembro de 2007, totalizando 20 consultas fisioterapêuticas. Após esse período, era aplicado um novo questionário SF-36 para, então, serem comparados aos resultados. Os resultados foram comparados utilizando-se o teste não paramétrico Wilcoxon, com nível de significância de 0,05 .

\section{Resultados}

Do total da amostra, oito pacientes (30\%) eram do sexo feminino e 19 (70\%) do sexo masculino. A média de idade foi de $51 \pm 10,5$ anos. 0 tempo de tratamento hemodialítico foi de $50 \pm 27,7$ meses. 0 índice de massa corpórea médio desses pacientes foi de $24,3 \pm 3,8$.

A principal patologia tratada, além da IRC, foi a hipertensão arterial sistêmica (HAS), presente em 14 casos (52\%), seguida da diabetes mellitus (26\%), das sequelas de nefrites (15\%) e de doença policística renal $(7 \%)$.

A capacidade funcional dos pacientes antes do tratamento fisioterapêutico alcançou média de $62 \pm$ 25. Após a aplicação do protocolo, a média subiu para $71 \pm 22$, sendo essa diferença considerada estatisticamente significativa $(\mathrm{p}=0,016)$ (Figura 1).

A limitação por aspectos físicos apresentada pelos pacientes antes da atuação fisioterapêutica atingiu uma média de $51 \pm 46$, aumentando tal média para $60 \pm 43$, porém, não sendo essa diferença considerada significativa após o tratamento fisioterapêutico $(\mathrm{p}=0,11)$ (Figura 2).

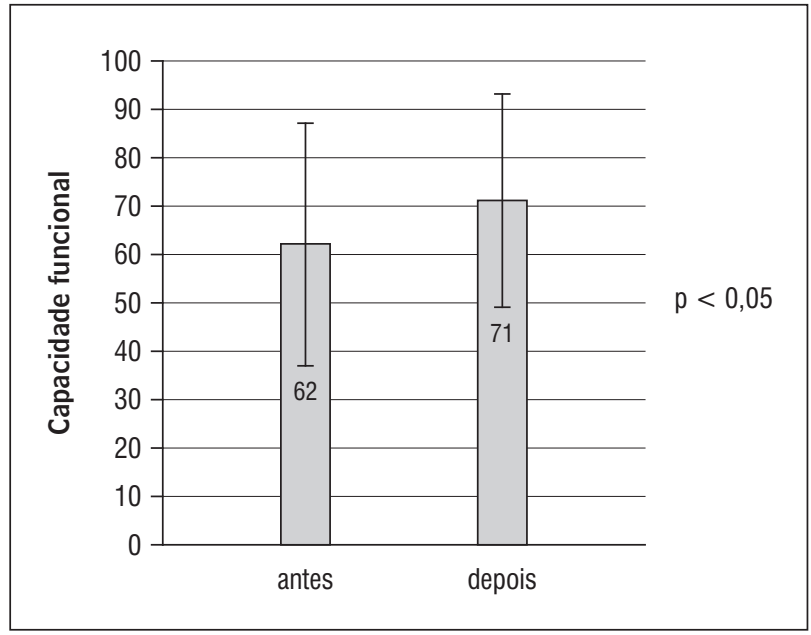

Figura 1 - Média e desvio-padrão da capacidade funcional dos pacientes antes e depois do protocolo fisioterapêutico $(n=27 ; p<0,05)$

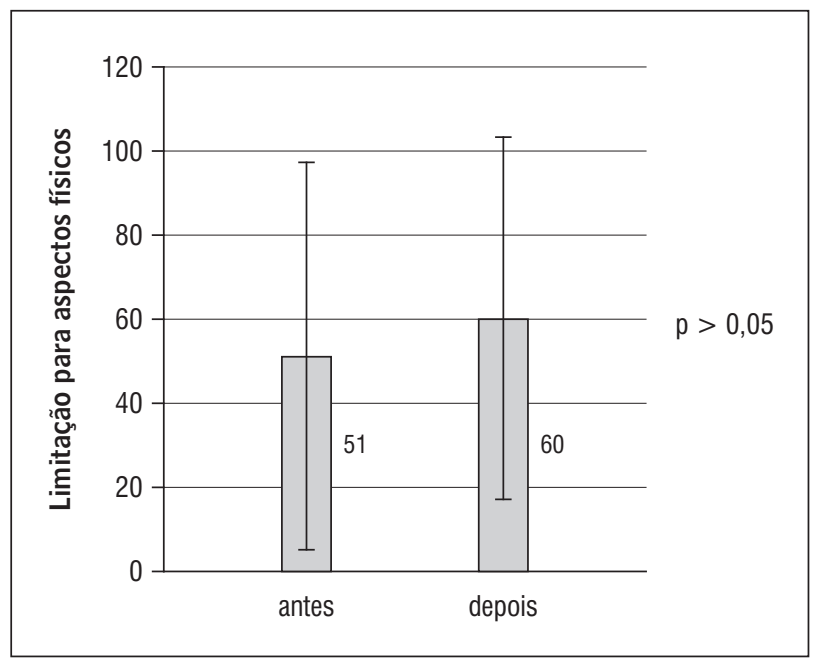

Figura 2 - Média e desvio-padrão da limitação por aspectos físicos dos pacientes antes e depois do protocolo fisioterapêutico $(n=27 ; p>0,05)$

Anteriormente ao tratamento, foi observado que dez pacientes relataram cãibras musculares, principalmente em membros inferiores. Após a intervenção, esse número diminuiu para apenas quatro.

0 nível de dor dos pacientes antes do tratamento fisioterapêutico teve média de $61 \pm 26$, elevando-se significativamente após a atuação fisioterapêutica $(75 \pm 29)(p=0,01)$ (Figura 3).

Inicialmente, o estado geral de saúde desses indivíduos teve média de $51 \pm 26$, não se alterando após o período da aplicação do protocolo de fisioterapia $(58 \pm 24)(p=0,12)$ (Figura 4). 


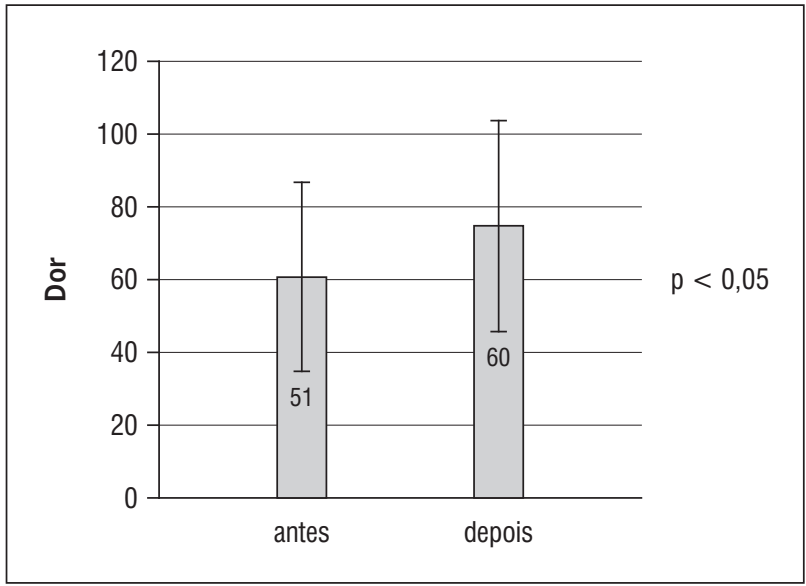

Figura 3 - Média e desvio-padrão do nível de dor dos pacientes antes e depois do protocolo fisioterapêutico $(n=27 ; p<0,05)$

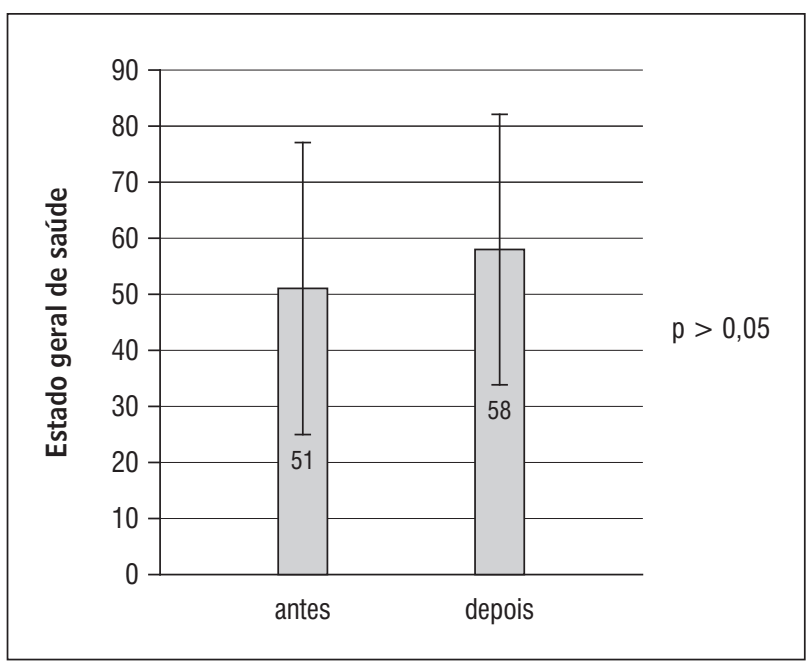

Figura 4 - Média e desvio-padrão do estado geral de saúde dos pacientes antes e depois do protocolo fisioterapêutico $(n=27 ; p>0,05)$

A vitalidade desses pacientes, antes da terapia, foi de $53 \pm 24$, elevando-se significativamente para $65 \pm 19$ após a intervenção fisioterapêutica ( $\mathrm{p}=$ 0,02 ) (Figura 5).

Quanto à limitação por aspectos sociais, antes da terapia alcançou-se média de $63 \pm 30$ e, após, passou-se para $70 \pm 24$, porém, tal diferença não foi considerada estatisticamente significativa $(\mathrm{p}=$ 0,19) (Figura 6).

A limitação por aspectos emocionais teve média de $53 \pm 42$. Tal média passou para $65 \pm 41$, não sendo essa alteração considerada significativa ( $p=$ 0,12 ) (Figura 7).

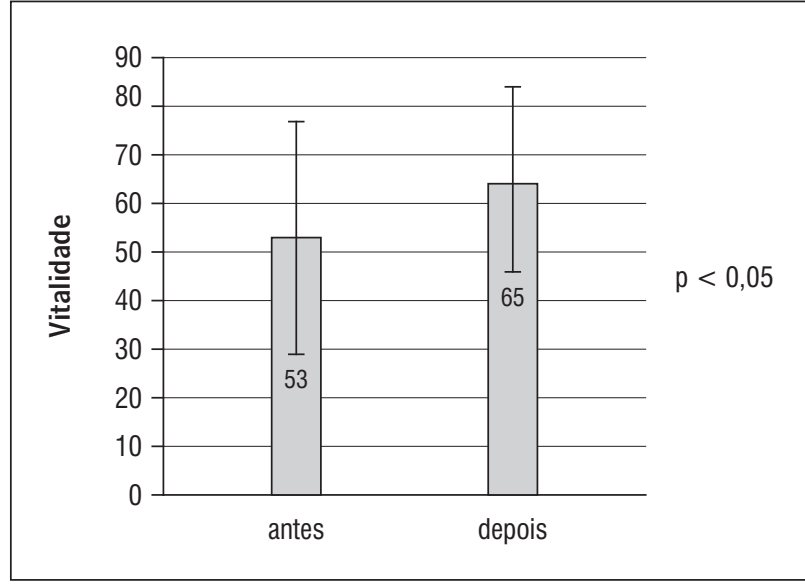

Figura 5 - Média e desvio-padrão da vitalidade dos pacientes antes e depois do protocolo fisioterapêutico $(\mathrm{n}=$ $27 ; p<0,05)$

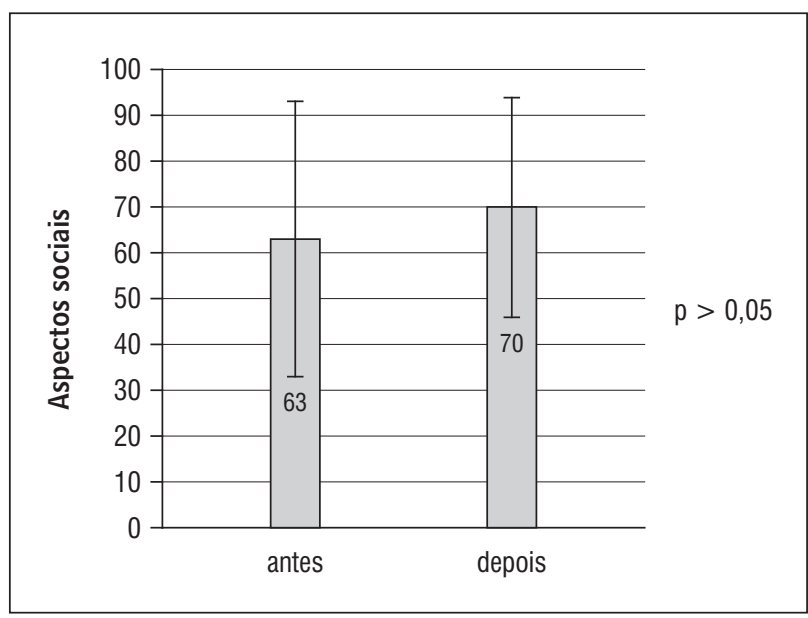

Figura 6 - Média e desvio-padrão dos aspectos sociais dos pacientes antes e depois do protocolo fisioterapêutico $(n=27 ; p>0,05)$

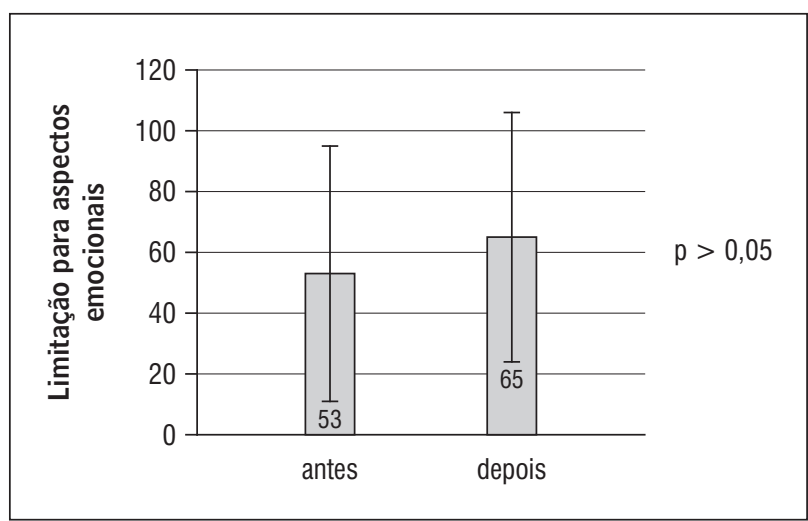

Figura 7 - Média e desvio-padrão da limitação por aspectos emocionais dos pacientes antes e depois do protocolo fisioterapêutico $(n=27 ; p>0,05)$ 
Por fim, o nível de saúde mental desses indivíduos elevou-se, após a intervenção, de uma média de $65 \pm$ 25 para $76 \pm 21$, sendo essa diferença considerada significativa $(\mathrm{p}=0,002)$ (Figura 8 ).

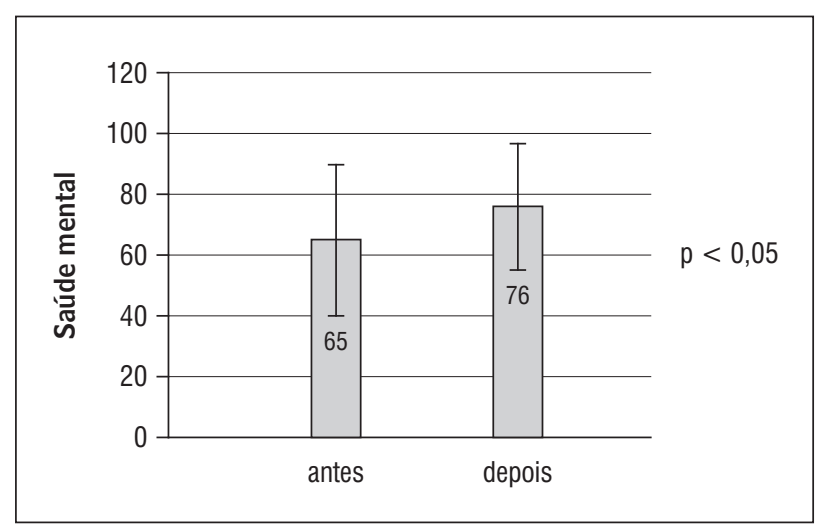

Figura 8 - Média e desvio-padrão da saúde mental dos pacientes antes e depois do protocolo fisioterapêutico $(n=27 ; p<0,05)$

\section{Discussão}

Tem crescido o interesse dos profissionais da saúde em mensurar a qualidade de vida dos portadores de IRC $(25,29,30)$, pois existe uma correlação direta entre o nível de qualidade de vida e a sobrevida $(17,18,25)$.

$\mathrm{Na}$ avaliação inicial, constatou-se comprometimento de todas as dimensões analisadas e os menores valores foram em limitação por aspectos físicos, estado geral de saúde, vitalidade e limitação por aspectos emocionais, o que já foi indicado pela literatura $(8,28)$.

Vários estudos (17-19) haviam apontado correlação negativa entre a idade dos pacientes e os seguintes domínios: capacidade funcional, limitação por aspectos físicos, dor, estado geral de saúde, vitalidade e aspectos sociais. No presente estudo foi possível constatar tal realidade, porém, não houve qualquer correlação entre idade e aspectos sociais. Não houve, também, diferença significativa de acordo com o sexo, o que já foi relatado por Martins e Santos $(8,16)$.

Com relação ao tempo de tratamento hemodialítico, foram encontradas algumas divergências. Santos (6), que avaliou o nível de qualidade de vida de 93 portadores de IRC em um período de 12 meses de hemodiálise, constatou correlação positiva entre aspectos emocionais desses indivíduos e o tempo de tratamento hemodialítico, o que incluiu adaptação psicológica à doença e à rotina de tratamento. No entanto, no atual trabalho, os resultados indicaram o oposto, corroborando, portanto, com Martins (8) e Castro (28). Deve-se ainda considerar que existem outros fatores, observados nesta amostra, que podem influenciar no componente emocional do paciente, tais como: número de intercorrências, problemas familiares ou no trabalho e presença de algum distúrbio depressivo preexistente. A relação dessas variáveis sobre o componente emocional ainda não foi mencionada em qualquer outro artigo da literatura.

Após o tratamento fisioterapêutico, constatou-se melhora significativa da capacidade funcional e do nível de dor nos participantes da pesquisa, o que já foi indicado pela literatura (13). Os pacientes com IRC queixam-se frequentemente de desconforto e/ ou dor, principalmente nas regiões lombar e cervical. Esse fato pode ser explicado por vários motivos, como: presença de distúrbios musculoesqueléticos $(9,15,16,21)$, permanência na posição "sentado" e "estático", má postura do paciente ao sentar e cadeiras padronizadas que, muitas vezes, não respeitam as individualidades ergonômicas de cada paciente (2).

Houve boa aderência dos pacientes ao tratamento proposto. Soares et al. (27) relatam que a taxa de aderência dos pacientes ao programa de reabilitação, durante a hemodiálise, é muito maior quando comparada a do programa ambulatorial, provavelmente porque, quando realizado durante a diálise, o exercício não tome tanto tempo como um programa formal realizado em outro turno.

Magalhães (2) citou que os exercícios de alongamento muscular são benéficos, pois devolvem aos músculos seu comprimento e elasticidade normais, o que pode ser muito útil na redução da incidência de cãibras. Com base nisso, nesta pesquisa foi observada a diminuição do número de pacientes com cãibras após a intervenção fisioterapêutica.

A cãibra é uma contração muscular forte e involuntária que causa dores nas regiões do corpo em que ocorre (2). Sua patogênese não está totalmente elucidada, mas está provavelmente relacionada à ultrafiltração rápida, à hiponatremia e à hipotensão (15). Magalhães (2), ao pesquisar a atuação da fisioterapia em 13 pacientes renais crônicos, comprovou que houve melhora significativa na incidência de cãibras, corroborando com os dados desta pesquisa. Os exercícios de fortalecimento muscular auxiliam na manutenção da tensão normal do músculo e no 
retorno venoso, atenuando a rápida perda de líquidos que a hemodiálise promove (2).

A fraqueza muscular é uma complicação frequente da IRC $(12,14,15)$. Sua etiologia não está totalmente esclarecida, porém, alguns fatores de risco podem ser citados, como: deficiência de carnitina, desnutrição, miopatias, atrofias musculares, excesso e toxicidade do paratormônio (PTH), toxinas urêmicas e deficiência de vitamina $\mathrm{D}(11,15)$. Ocorre, também, atrofia de ambas as fibras, principalmente as do tipo II (11).

Logo, pode-se afirmar a importância dos exercícios de fortalecimento para minimizar essa perda de massa muscular, além de promover a força necessária para que o indivíduo exerça suas AVDs com menor esforço (2).

Observou-se, também, melhora da vitalidade e da saúde mental desses indivíduos. Provavelmente, quando o indivíduo consegue realizar suas AVDs com menor esforço e/ou dor, sente-se menos cansado e mais motivado. Esse fato repercute sobre o estado emocional.

É importante observar que os pacientes com menor tempo em terapia hemodialítica apresentaram melhores resultados. Logo, se faz necessária uma pesquisa com populações mais homogêneas.

Coelho (11) e Peres (24) afirmaram que exercícios físicos proporcionam redução da pressão arterial sistólica (PAS) e melhora da função cardiovascular. Contudo, neste estudo não foi constatada a melhora da PA nos pacientes, uma vez que não houve nenhuma estimulação física aeróbica, proporcionando uma melhor hemodinâmica, conforme demonstrado por Magalhães, Coelho e Peres $(2,11,24)$.

\section{Conclusão}

De acordo com os resultados obtidos, os programas de reabilitação física são benéficos para a melhora do estado geral e da qualidade de vida dos pacientes renais crônicos. Sugere-se, portanto, abrir novas perspectivas e estimular a realização de novos estudos nessa área, com um número maior de pacientes para confirmação desses achados.

\section{Agradecimentos}

Gostaríamos de agradecer aos nossos orientadores, Tânia e Edison, à profa. Ana Tereza e a todos os pacientes e funcionários do Instituto do Rim do Paraná, que muito contribuíram na construção deste trabalho.

\section{Referências}

1. Bastos MG, Carmo WB, Abrita RR, Almeida EC, Mafra D, Costa DMN, et al. Doença renal crônica: problemas e soluções. J Bras Nefrol. 2004;26(4):202-15.

2. Ajzen H, Schon N. Nefrologia. São Paulo: Manole; 2002.

3. Barros E, Manfro RC, Thomé FS, Gonçalves LFS. Nefrologia: rotinas, diagnóstico e tratamento. 3a ed. Porto Alegre: Artmed; 2006.

4. Costanzo LS. Fisiologia renal. In: Costanzo LS. Fisiologia. 2a ed. Rio de Janeiro: Elsevier; 2004. p. 223-309.

5. Santos PR, Pontes LRSK. Mudança do nível de qualidade de vida em portadores de insuficiência renal crônica terminal durante seguimento de doze meses. Rev Assoc Med Bras. 2007;53(4):329-34.

6. Sociedade Brasileira de Nefrologia. Censo da SBN, 2007. [acesso em 21 jul. 2009]. Disponível em: www.sbn.org.br

7. Magalhães HG, Pinto TA, Reboredo MM, Fonseca FD, Almeida PC. Análise da eficácia do atendimento fisioterapêutico em pacientes com doença renal crônica em hemodiálise. $2^{\underline{0}}$ Congresso Brasileiro de Extensão Universitária; 12-15 set. 2004. Belo Horizonte, MG: UFMG; 2004.

8. Martins MRI, Cesarino CB. Qualidade de vida de pessoas com doença renal crônica em tratamento hemodialítico. Rev Latino-Am Enferm. 2005;13(5):670-6.

9. Lima JJG, Fonseca JA, Godoy AD. Dialysis, time and death: comparisons of two consecutive decades among patients treated at the same Brasilian dialysis center. Braz J Med Biol Res. 1999; 32 (3):289-95.

10. Coelho DM, Castro AM, Tavares HA, Abreu PCB, Glória RR, Duarte MH, et al. Efeitos de um programa de exercícios físicos no condicionamento de pacientes em hemodiálise. J Bras Nefrol. 2006;28(3):121-7.

11. Cury JL, Togoe EB, Rossoni LA. Alterações da força muscular respiratória em indivíduos com insuficiência renal crônica. 13은 Simpósio Internacional de Fisioterapia Respiratória e Fisioterapia em Terapia Intensiva; set. 6-9, 2006; Curitiba, PR. Associação Brasileira de Pesquisa e Pós-Graduação em Fisioterapia; 2006. 
12. Kovelis D, Pitta F, Probst VS, Peres CPA, Delfino VDA, Mocelin AJ, et al. Função pulmonar e força muscular respiratória em pacientes com doença renal crônica submetidos à hemodiálise. J Bras Pneumol. 2008; 34(11):907-12.

13. Gomes CP, Silva MIB, Duarte MEL, Dorigo D, Lemos CCS, Bregman R. Bone disease in patients with chronic kidney disease under conservative management. São Paulo Med J. 2005;123(2):83-7.

14. Vieira WP, Gomes KWP, Frota NB, Andrade JECB, Vieira RMRA, Moura FEA, et al. Manifestações musculoesqueléticas em pacientes submetidos à hemodiálise. Rev Bras Reumatol. 2005;45(6):357-64.

15. Braz AS, Duarte ALBP. Manifestações musculoesqueléticas nos pacientes em programa de hemodiálise. Rev Bras Reumatol. 2003;43(4):223-31.

16. Santos PR. Relação do sexo e da idade com nível de qualidade de vida em renais crônicos hemodialisados. Rev Assoc Med Bras. 2006;52(5):356-9.

17. Bohlke M, Nunes DL, Marini SS, Kitamura C, Andrade M, Von-Gysel MPO. Predictors of quality of life among patients on dialysis in southern Brazil. São Paulo Med J. 2008;126(5):252-6.

18. Souza FF, Cintra FA, Gallani MCBJ. Qualidade de vida e severidade da doença em idosos renais crônicos. Rev Bras Enferm. 2005;58(5):540-4.

19. Duarte MEL, Peixoto ALP, Pacheco AS, Peixoto AV, Rodriguez RD, Lugon JR, et al. The spectrum of bone disease in 200 chronic hemodialysis patients: a correlation between clinical, biochemical and histological findings. Rev Paul Med. 1998;116(5):1790-7.

20. Lugon JR, Anfré MB, Duarte MEL, Rembold SM, Cruz EAS. Effects of in - center daily hemodialysis upon mineral metabolism and bone disease in end - stage renal disease patients. São Paulo Med J. 2001;119(3):105-9.

21. Henn EB, Paiva DN, Albuquerque IM. Estudo comparativo da função pulmonar de pacientes com insuficiência renal crônica (IRC) pré e pós-sessão de hemodiálise. 13ํㅗำ Simpósio Internacional de Fisioterapia Respiratória e Fisioterapia em Terapia Intensiva; set. 6-9, 2006; Curitiba, PR. São Carlos: Associação Brasileira de Pesquisa e Pós-Graduação em Fisioterapia; 2006.

22. Lima JJG. Practical ways to deal with the high burden of cardiovascular disease in hemodialysis patients. São Paulo Med J. 2006; 124 (1):36-41.
23. Barberato SH, Pecoits Filho R. Valor prognóstico do índice do volume do átrio esquerdo em pacientes em hemodiálise. Arq Bras Cardiol. 2007; 88(6):643-50.

24. Higa K, Kost MT, Soares DM, Morais MC, Polins BRG. Qualidade de vida de pacientes portadores de insuficiência renal crônica em tratamento de hemodiálise. Acta Paul Enferm. 2008; 21 (n. spe):203-6.

25. Peres CPA, Kovelis D, Arnaut AC, Bonomo C, Ferrari R, Santos GCCO, et al. Efeitos de um programa de exercícios físicos em pacientes com insuficiência renal crônica em hemodiálise. 13ํㅗํ Simpósio Internacional de Fisioterapia Respiratória e Fisioterapia em Terapia Intensiva; set. 6-9, 2006; Curitiba, PR. São Carlos: Associação Brasileira de Pesquisa e Pós-Graduação em Fisioterapia; 2006.

26. Reboredo MM, Henrique DMN, Bastos MG, Paula RB. Exercício físico em pacientes dialisados. Rev Bras Med Esporte. 2007;13(6):427-30.

27. Soares A, Zehetmeyer M, Rabuske M. Atuação da fisioterapia durante a hemodiálise visando a qualidade de vida do paciente renal crônico. Rev de Saúde UCPEL. 2007;1(1):7-12.

28. Ciconelli RM, Ferraz MB, Santos W, Meinão I, Quaresma MR. Tradução para a língua portuguesa e validação do questionário genérico de avaliação de qualidade de vida SF-36 (Brasil SF-36). Rev Bras Reumatol. 1998;39(3)143-50.

29. Castro M, Caiuby AVS, Draibe AS, Canziani MEF. Qualidade de vida de pacientes com insuficiência renal crônica em hemodiálise avaliada através do instrumento genérico SF-36. Rev Assoc Med Bras. 2003; 49(3):245-9.

30. Duarte PS, Miyaziaki MCOS, Ciconelli RM, Sesso R. Tradução e adaptação cultural do instrumento de avaliação de qualidade de vida para pacientes renais crônicos (KDQOL-SF). Rev Assoc Med Bras. 2003; 49(4):375-81.

Recebido: 09/07/2010

Received: 07/09/2010

Aprovado: 24/09/2010

Approved: 09/24/2010 